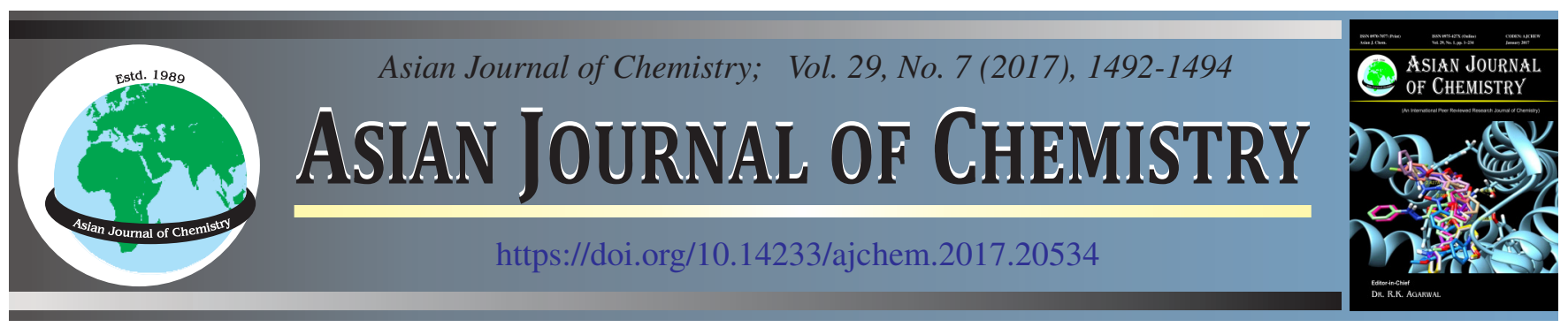

\title{
Synthesis and Thermal Properties of Copper Nanoparticles
}

\author{
Jyoti Chaudhary ${ }^{1}$, Giriraj TAILOR ${ }^{1}$, DeEPak Kumar ${ }^{2}$ and AJit Joshi, ${ }^{3}$,
}

${ }^{1}$ Department of Polymer Science, Mohanlal Sukhadia University, Udaipur-313 001, India

${ }^{2}$ Department of Chemistry, Indian Institute of Technology Jodhpur, Jodhpur-342 011, India

${ }^{3}$ Department of Chemistry, Mewar University, Gangrar-312 901, India

*Corresponding author: E-mail: ajitjoshi2k2@gmail.com; ajitj@mewaruniversity.co.in

\begin{abstract}
Copper nanoparticles have been synthesized by simple chemical precipitation method and showed versatile thermal, mechanical, magnetic and electrical properties. Copper nanoparticles have been synthesized in following two steps, in step- $1^{\text {st }}$, synthesis of polymer metal complex and in $2^{\text {nd }}$ step formation of copper nanoparticles. Polymer metal complex is confirmed by IR spectroscopy and XRD confirmed

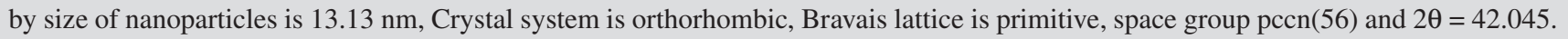
Thermal stability is confirmed by TGA and the compound is very slow (rate of $3 \%$ ) loss in weight per $100{ }^{\circ} \mathrm{C}$ increase in the temperature up to $900{ }^{\circ} \mathrm{C}$. It shows that $70 \%$ thermal stability of the compound at $900{ }^{\circ} \mathrm{C}$. Endothermic nature of copper nanoparticles has been confirmed by DSC.
\end{abstract}

Keywords: Copper-nanoparticle, Thermal stability, Orthorhombic, Bakelite.

\section{INTRODUCTION}

Nanomaterial is a mesmerizing material that is found in many applications in the field of basic and applied research. Copper nanoparticles with high specific surface to volume area have been widely studied. The copper nanoparticles have special physico-chemical characteristic which include catalytic activity, electronic properties and antimicrobial activity [1]. Nanoparticles have received considerable interest due to their good thermal, optical and electrical properties. Copper oxide is a semiconducting compound and its structure is a monoclinic. Copper compounds exhibit a range of potentially useful physical properties such as high temperature superconductivity and spin dynamic $[2,3]$. As in important p-type semiconductor copper oxide has found many diverse applications. Copper nanoparticles have been assumed cost effective as compared to noble metals like Ag, Au and Pt. They are potentially applied in the fields of catalysis [4], conductive inks [5] and fluids [6]. The synthesis of mono disperse, ultrafine metal nanoparticles have been potential application in photonics, catalysis, nanofluides, cooling fluids for electronic systems, Plasmonic photovoltaic and composite food packaging [7-14]. There are different methods of synthesis of copper nanoparticles including thermal decomposition [15], solid liquid discharge [16], quick precipitation [17] metal vapour synthesis [18], chemical reduction [19], vacuumed vapour deposition [20], radiation method [21], micro-emulsion techniques [22], polyol processes [23] and laser ablation [24]. Major limitations in the synthesis of their ease of oxidation [25]. Chemical precipitation method is one of the most convenient methods for the synthesis of metallic nanoparticles because this synthesis is simple, shape and size of nanoparticles can be controlled. Copper nanoparticles have been prepared by simple chemical precipitation method and characterized by infrared spectroscopy, scanning electron microscope and thermal gravimetric analysis.

\section{EXPERIMENTAL}

All the chemicals were used of AR grade, formaldehyde and phenolfrom [Central Drug House (P) Ltd.], glacial acetic acid and hydrochloride acid (Fisher scientific). Metal solution has been prepared by dissolving appropriate amount of copper(II) chloride in distilled water.

\section{Synthesis of copper nanoparticles}

Set- ${ }^{\text {st }}$ : Synthesis of polymer metal complex: In 250 $\mathrm{mL}$ beaker taken of $10 \mathrm{~g}$ phenol and $20 \mathrm{~mL}$ of formaldehyde after continuous $15 \mathrm{~min}$ stirring, then $25 \mathrm{~mL}$ of glacial acetic acid was added drop by drop with maintained temperature. Few drops of $\mathrm{HCl}$ was also added. After stirrer few minute, light pink colour bakelite compound was formed. In another beaker freshly prepared $1 \mathrm{~N}$ copper solution was taken. Added drop by drop $15 \mathrm{~mL}$ of $1 \mathrm{~N}$ metal ion solution then the reaction 
mixture was continued stirrer for $30 \mathrm{~min}$ then heated at $45^{\circ} \mathrm{C}$ for $1 \mathrm{~h}$ on heating mental. After heating the polymer composite was formed. The solid sample had been purified by the washing with distilled water solution. The excess metal ion and impurities on the sample purified by washing. Then it was kept into the desiccators for drying.

Set-2 ${ }^{\text {nd }}$ : Synthesis of copper nanoparticles: In order to get nanoparticles of polymer metal complex decomposition takes place at $850{ }^{\circ} \mathrm{C}$ for $30 \mathrm{~min}$ and purification done in following steps.

\section{Purification of copper nanoparticles}

Step-1 ${ }^{\text {st }}$ : Remove volatile impurity: At the time of decomposition many volatile impurities were separated and nanoparticles become free from these impurities.

Step-2 ${ }^{\text {nd }}$ : Remove metallic impurities: For removal of the metallic ions, nanoparticles were kept in $12 \mathrm{~N} \mathrm{HCl}$ solution for $24 \mathrm{~h}$. Then they were centrifuged and washed with distilled water till hydrochloric acid was completely removed.

\section{RESULTS AND DISCUSSION}

${ }^{1}$ H NMR spectra: The analysis of NMR spectra of polymer metal complex indicate three types of proton present in sample. Peak at $3.524 \mathrm{ppm}$ shows that phenolic protons present in compounds, peak at $2.471 \mathrm{ppm}$ it represent that the benzylic proton is present in polymer metal complex, at 6.744 ppm it represent aromatic proton present in polymer metal complex. Multiplates at 6.744, 3.695 and $2.471 \mathrm{ppm}$, it represents that the formed complex polymerized substances have been formed.

IR spectra: The IR Spectra analysis of polymer metal complex are given in Table-1.

\begin{tabular}{cc}
\multicolumn{3}{c}{ TABLE-1 } \\
IR SPECTRA \\
\hline Prominent absorption band $\left(\mathrm{cm}^{-1}\right)$ & Functional group \\
\hline 3278 & OH stretching (broad peak) \\
$3007-2921$ & C-H stretching (aromatic) \\
1173 & C-O stretching \\
\hline
\end{tabular}

X-RD characterization: Orthorhombic copper nanoparticles have been determined using XRD technique. Other use of XRD technique is to evaluate the particle size using Scherrer equation:

$$
\mathrm{D}=\mathrm{K} \lambda /(\mathrm{B} \cos \theta)
$$

where $\mathrm{D}$ is the mean size of crystallites (nm), K is crystallite shape factor a good approximation is $0.9, \lambda$ is $\mathrm{X}$-ray wavelength, $\mathrm{B}$ is full width at half the maximum (FWHM) in radians of the X-ray diffraction peak and $\theta$ is the Bragg angle. Different copper nanostructures sizes were obtained using wet chemical precipitation method. By applying Debye-Scherrer equation to the obtained XRD pattern (Fig. 1) of the copper nanoparticles, the average nanoparticles size was found to be 13.13 $\mathrm{nm}$ and bravais lattice is primitive and space group is $\operatorname{pccn}(56)$ and $2 \theta=42.045$.

TGA and DSC: Thermal properties of copper nanoparticles were characterized using thermogravimetric analysis (TGA). The TGA thermograph predicts the $30 \%$ mass decom-

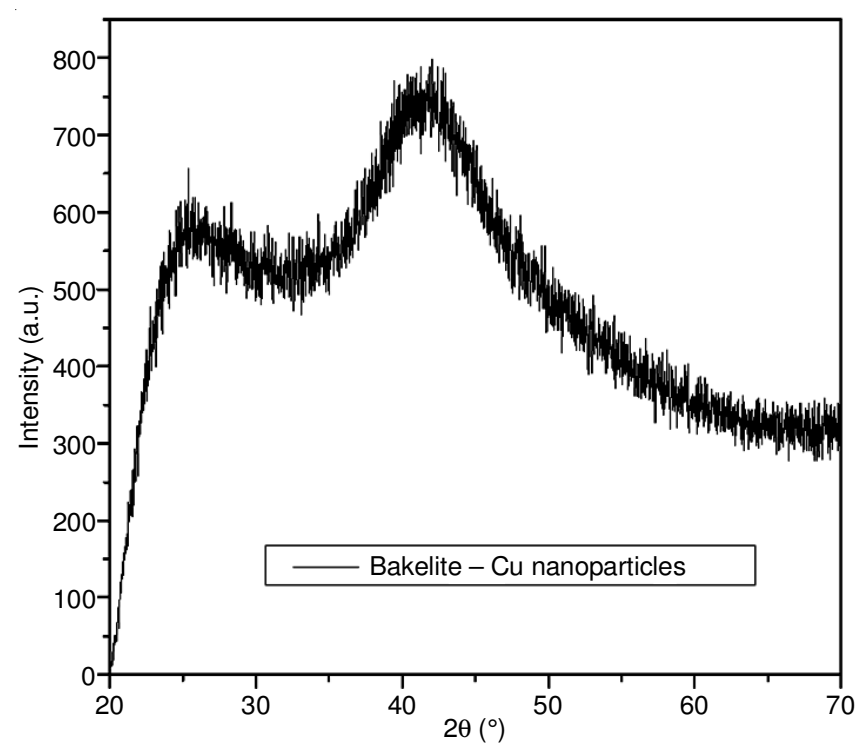

Fig. 1. XRD spectra of copper nanoparticles

position of composite from $29-900{ }^{\circ} \mathrm{C}$. The TGA graph shows at a heating up to $100^{\circ} \mathrm{C} / \mathrm{min}$. The graph shows little loss in a sample with increased temperature. The decomposition of copper nanoparticles takes place at very slower rate of $3 \%$ loss in weight per $100{ }^{\circ} \mathrm{C}$ increase in the temperature up to $900{ }^{\circ} \mathrm{C}$. It shows that $70 \%$ thermal stability of the compound at $900{ }^{\circ} \mathrm{C}$ (Fig. 2). The isothermal behaviour of copper nanoparticles has been investigated using DSC technique over the temperature range $25-900^{\circ} \mathrm{C}$ in ambient air. DSC curve (Fig. 3) of copper nanoparticles shows the large endothermic peak at $609.61{ }^{\circ} \mathrm{C}$.

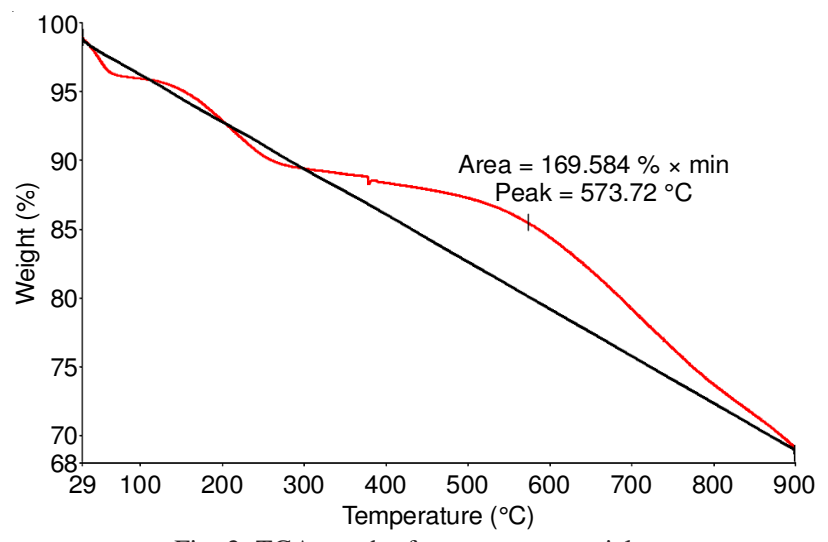

Fig. 2. TGA graph of copper nanoparticles

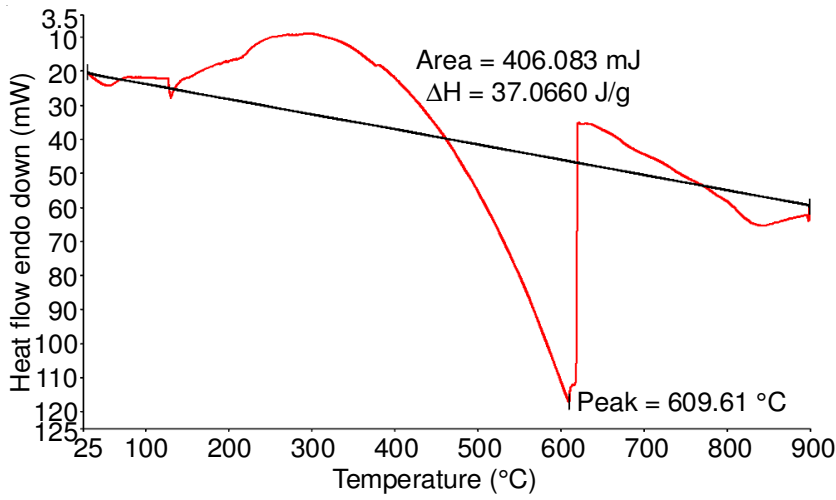

Fig. 3. DSC graph of copper nanoparticles 


\section{Conclusion}

Copper nanoparticles have been synthesized by the chemical precipitation method. The complex formation is characterized by infrared spectroscopy and NMR spectroscopy. The crystallinity of the synthesized nanoparticles is confirmed by XRD and result revealed that size of copper nanoparticles were of $13.13 \mathrm{~nm}$. Thermal studies confirms the high thermal stability of and endothermic nature of nanoparticles.

\section{ACKNOWLEDGEMENTS}

The authors are thankful to The Head, Department of Polymer Science, M.L. Sukhadia University, Udaipur and Department of Chemistry, Mewar University, Gangrar, India for providing laboratory facilities. Thanks are also due to IIT Jodhpur, India for spectral analysis.

\section{REFERENCES}

1. T. Jumaa, M. Chasib, M.K. Hamid and R. Al-Haddad, Nanosci. Nanotechnol. Res., 2, 1 (2014);

https://doi.org/10.12691/nnr-2-1-1.

2. R.J. Cava, Science, 247, 656 (1990); https://doi.org/10.1126/science.247.4943.656.

3. J.M. Tranquada, B.J. Sternlieb, J.D. Axe, Y. Nakamura and S. Uchida, Nature, 375, 561 (1995); https://doi.org/10.1038/375561a0.

4. P. Singh, A. Katyal, R. Kalra and R. Chandra, Tetrahedron Lett., 49, 727 (2008);

https://doi.org/10.1016/j.tetlet.2007.11.106.

5. H.S. Kim, S. Dhage, D.E. Shim and H.T. Hahn, Appl. Phys., A Mater. Sci. Process., 97, 791 (2009);

https://doi.org/10.1007/s00339-009-5360-6.

6. Y. Lee, J. Choi, K.J. Lee, N.E. Stott and D. Kim, Nanotechnology, 19, 415604 (2008); https://doi.org/10.1088/0957-4484/19/41/415604

7. J.Z. Zhang and C. Noguez, Plasmonics, 3, 127 (2008); https://doi.org/10.1007/s11468-008-9066-y.

8. C.E. Sabastine and G. Fanchini, Dissertation, The University of Western Ontario, Ontario, Canada (2012).

9. R. Kaur, C. Giordano, M. Gradzielski and S.K. Mehta, Chem. Asian J., 9, 189 (2014); https://doi.org/10.1002/asia.201300809.
10. M. Quinten, Optical Properties of Nanoparticle Systems: Mie and beyond, Wiley-VCH, Germany (2011).

11. P.K. Jain, X. Huang, I.H. El-Sayed and M.A. El-Sayed, Acc. Chem. Res., 41, 1578 (2008); https://doi.org/10.1021/ar7002804.

12. X.-F. Tang, Z.-G. Yang and W.-J. Wang, Colloids Surf. A Physicochem. Eng. Asp., 360, 99 (2010); https://doi.org/10.1016/j.colsurfa.2010.02.011.

13. B. Lee, Y. Kim, S. Yang, I. Jeong and J. Moon, Curr. Appl. Phys., 9, e157 (2009); https://doi.org/10.1016/j.cap.2009.03.008.

14. A.M. Stefan, Plasmonics: Fundamentals and Applications, Springer (2007).

15. E. Darezereshki and F. Bakhtiari, J. Min. Metall. Sect. B-Metall., 47, 73 (2011); https://doi.org/10.2298/JMMB1101073D.

16. W.T. Yao, S.H. Yu, Y. Zhou, J. Jiang, Q.S. Wu, L. Zhang and J. Jiang, J. Phys. Chem. B, 109, 14011 (2005); https://doi.org/10.1021/jp0517605.

17. J. Zhu, D. Li, H. Chen, X. Yang, L. Lu and X. Wang, Mater. Lett., 58, 3324 (2004); https://doi.org/10.1016/i.matlet.2004.06.031.

18. G. Vitulli, M. Bernini, S. Bertozzi, E. Pitzalis, P. Salvadori, S. Coluccia and G. Martra, Chem. Mater, 14, 1183 (2002); https://doi.org/10.1021/cm011199x

19. X. Cheng, X. Zhang, H. Yin, A. Wang and Y. Xu, Appl. Surf. Sci., 253, 2727 (2006); https://doi.org/10.1016/j.apsusc.2006.05.125.

20. Z. Liu and Y. Bando, Adv. Mater., 15, 303 (2003); https://doi.org/10.1002/adma.200390073.

21. S.S. Joshi, S.F. Patil, V. Iyer and S. Mahumuni, Nanostruct. Mater, 10, 1135 (1998); https://doi.org/10.1016/S0965-9773(98)00153-6.

22. D.H. Chen and S.H. Wu, Chem. Mater, 12, 1354 (2000); https://doi.org/10.1021/cm991167y.

23. B.K. Park, D. Kim, S. Jeong, J. Moon and J.S. Kim, Thin Solid Films, 515, 7706 (2007) https://doi.org/10.1016/j.tsf.2006.11.142.

24. H.L. Aye, S. Choopun and T. Chairuangsri, Adv. Mater. Res., 93-94, 83 (2010); https://doi.org/10.4028/www.scientific.net/AMR.93-94.83.

25. S.H. Wu and D.H. Chen, J. Colloid Interface Sci., 273, 165 (2004); https://doi.org/10.1016/j.jcis.2004.01.071. 